\title{
Local tumor progression following lipiodol-based targeted chemoembolization of hepatocellular carcinoma: a retrospective comparison of miriplatin and epirubicin
}

\author{
This article was published in the following Dove Press journal: \\ Cancer Management and Research \\ 18 April 2012 \\ Number of times this article has been viewed
}

Jin Iwazawa'

Shoichi Ohue ${ }^{2}$

Naoko Hashimoto'

Takashi Mitani'

'Department of Radiology, Nissay Hospital, Nishiku, Osaka, ${ }^{2}$ Department of Radiology, Komatsu Hospital, Neyagawa, Japan
Correspondence: Jin Iwazawa Nissay Hospital, Department of Radiology, 6-3-8 Itachibori, Nishiku, Osaka 550-0012, Japan

Tel +8I 66543358 I

Fax +81665326482

Email iwazawa.jin@nissay-hp.or.jp
Purpose: We aimed to compare the local control rates between miriplatin and epirubicin in lipiodol-based transarterial chemoembolization (TACE) for hepatocellular carcinoma (HCC). Patients and methods: Patients who underwent targeted TACE using miriplatin (47 patients, 66 lesions) or epirubicin (64 patients, 79 lesions) as the sole therapy were enrolled. The local control rates were compared using the Kaplan-Meier estimator with the log-rank test. The patient and tumor parameters were subjected to univariate and multivariate analyses using the Cox proportional hazards model.

Results: The overall local recurrence rates were $39.3 \%$ and $31.6 \%$ for the miriplatin and epirubicin groups, respectively. The local control rate was significantly higher in the epirubicin group than in the miriplatin group $(P<0.001)$. The local control rates at 6 months and 1 year were $70.7 \%$ and $44.8 \%$ for the miriplatin group and $83.4 \%$ and $69.2 \%$ for the epirubicin group, respectively. Multivariate analysis showed that the serum $\alpha$-fetoprotein level $\geq 20 \mathrm{ng} / \mathrm{mL}$ (hazard ratio 2.96; $P<0.001$ ), miriplatin usage (hazard ratio 2.53; $P=0.002$ ), and Child-Pugh class $\mathrm{B}$ (hazard ratio $1.89 ; P=0.042$ ) affected local progression.

Conclusion: Lipiodol-based targeted TACE using miriplatin had inferior local control rates as compared to epirubicin in patients with HCC.

Keywords: chemoembolization, miriplatin, epirubicin, liver, neoplasms

\section{Introduction}

Transarterial chemoembolization (TACE) is an established local therapy for managing unresectable hepatocellular carcinoma (HCC) in patients with advanced cirrhosis and confers significant survival benefits. ${ }^{1,2}$ Iodized oil is usually used as a carrier of most anticancer agents to achieve preferential uptake and persistent deposit of the chemotherapeutic agent within the HCC nodules in conventional TACE. Most anticancer agents used in TACE are hydrophilic and usually prepared as emulsions mixed with iodized oil before intraarterial administration. ${ }^{3}$ These hydrophilic agents may cause rapid release of the active anticancer components in the bloodstream after they accumulate in the target lesions; therefore, these agents may not be expected to yield persistent and sufficient anticancer drug levels in the plasma against tumor growth. ${ }^{4}$

Miriplatin (Miripla; Dainippon Sumitomo Pharma, Osaka, Japan) is a thirdgeneration platinum derivative and has been developed recently for transarterial treatment of HCC..$^{5}$ Unlike other hydrophilic anticancer agents, miriplatin contains 
myristates as lipophilic side chains, which combine with the carrier ligand of platinum; therefore, it is easily dissolved in iodized oil without the need for emulsification. ${ }^{6}$ Following intra-arterial administration, the miriplatin-iodized oil suspension accumulates in the target tumor, and continuous antitumor effects caused by gradual release of active platinum compounds are expected..$^{6-8}$ In an early Phase II trial, 56\% of the patients treated with miriplatin via transarterial chemoinfusion therapy were shown to achieve a complete response without major adverse events. ${ }^{4}$ In a randomized late Phase II study in patients with unresectable HCC, the miriplatin suspension demonstrated similar therapeutic efficacy to a zinostatin stimalamer suspension; further, it caused less hepatic vascular injury than the latter. ${ }^{9}$ These clinical studies suggest that miriplatin is a promising alternative to conventional hydrophilic chemotherapeutic agents such as cisplatin or doxorubicin for treating unresectable HCC.

Miriplatin was approved for clinical use and covered by public health insurance in Japan in January 2010. Thereafter, we initiated the clinical use of miriplatin instead of epirubicin hydrochloride (Farmorubicin; Pfizer Japan, Tokyo, Japan) for every TACE procedure for treating $\mathrm{HCC}$ at our institute. In this study, we retrospectively compared the local tumor control rate of miriplatin-iodized oil suspension with that of epirubicin-iodized oil emulsion and evaluated the prognostic factors affecting local tumor progression in the targeted TACE for HCC.

\section{Patients and methods}

\section{Patients}

Consecutive patients with unresectable $\mathrm{HCC}$ who had received targeted TACE with miriplatin or epirubicin as the sole therapy between April 2008 and July 2011 at our institution were considered for inclusion in this study. Each patient was required to meet the following criteria: no previous treatment for the lesions under study, a Child-Pugh classification of A or B, total serum bilirubin level of $<3 \mathrm{mg} / \mathrm{dL}$, no portal venous thrombus in the main trunk, no previous history of chemotherapy with platinum derivatives, an interval of at least 4 weeks after the cessation of any previous anticancer therapy, segmental or more distal chemoembolization, and no more than three intrahepatic lesions.

Finally, 111 patients with $145 \mathrm{HCC}$ nodules were included in this study. The diagnosis of HCC was confirmed from previous imaging findings as well as by the elevated levels of serum tumor markers. Serum $\alpha$-fetoprotein level of $\geq 20 \mathrm{ng} / \mathrm{mL}$ and/or serum des-carboxy-prothrombin level of $\geq 40 \mathrm{mAU} / \mathrm{mL}$ were considered as positive tumor markers. Serum $\alpha$-fetoprotein levels were measured by latex photometric assay (LPIA 100; Mitsubishikasei, Tokyo, Japan) and serum des-carboxy-prothrombin levels were measured by the electrochemiluminescence immunoassay method (Picolumi PIVKA-II; Eisai, Tokyo, Japan). The detection limits for serum $\alpha$-fetoprotein and des-carboxy-prothrombin levels were $2 \mathrm{ng} / \mathrm{mL}$ and $5 \mathrm{mAU} / \mathrm{mL}$, respectively. The size and number of tumors were determined from the conebeam computed tomography (CT) images obtained during each TACE session. Because we replaced epirubicin with miriplatin for all TACE procedures at our institution from June 2010 onwards, 64 patients with 79 lesions received epirubicin treatment between April 2008 and June 2010 and another 47 patients with 66 lesions received miriplatin treatment between June 2010 and July 2011.

This study was carried out in accordance with the guidelines of our institutional review board, and written informed consents were obtained from all patients for TACE using miriplatin or epirubicin.

\section{Drug preparation}

The miriplatin-iodized oil suspension was prepared by dissolving $70 \mathrm{mg}$ of miriplatin into $4-5 \mathrm{~mL}$ of iodized oil (Lipiodol Ultrafluid; Terumo, Tokyo, Japan), while the epirubicin-iodized oil emulsion was prepared by dissolving $10 \mathrm{mg}$ epirubicin into $1 \mathrm{~mL}$ of iopamidol (Iopamiron 370; Bayer Schering Pharma, Osaka, Japan) and then mixing that into 1-2 $\mathrm{mL}$ of iodized oil. The maximum dose for a single TACE session was limited to $140 \mathrm{mg}$ for miriplatin and $50 \mathrm{mg}$ for epirubicin. The actual dose was determined based on the size and number of target tumors and the liver function of the patient.

\section{Transarterial chemoembolization}

All the TACE procedures were performed using the same angiographic system (Innova 3100; GE Healthcare, Waukesha, WI) by the same two interventional radiologists, who have more than 10 years' experience in hepatic vascular interventions. First, an appropriate microcatheter was coaxially inserted through a 4-F catheter via the femoral artery and placed into the tumor-supplying artery. Second, a cone-beam CT image was obtained by injecting iopamidol from the microcatheter to confirm whether the target tumor was actually located within the treatment area. After the tumor location was confirmed, each hepatic area containing the target tumors was embolized with gelatin particles (Gelpart; Nippon Kayaku, Tokyo, Japan) after infusion with the appropriate concentration of chemoth 
erapeutic agents. Administration of the drugs and gelatin particles was terminated when the tumor vessels were completely filled with the drugs and the tumor stain disappeared on angiographic imaging.

\section{Treatment evaluation}

Seven days after the TACE session, the initial iodized oil uptake was assessed by unenhanced CT by using a 16-multidetector CT scanner (Somatom Sensation; Siemens Medical Solutions, Forchheim, Germany). Uptake of the iodized oil was graded as good, fair, or poor by a single reader, on the basis of its accumulation in the tumor. The uptake was scored as good when complete and dense uptake occurred throughout the tumor, fair when inhomogeneous uptake occurred throughout the tumor, and poor when there was evidence of failure of uptake. Only lesions that were scored good or fair were included in the study.

Tumor progression was judged using triphasic contrastenhanced CT or magnetic resonance (MR) imaging. Follow-up CT/MR imaging was performed every 1-3 months, depending on previous imaging findings and the laboratory data. Recurrence was evaluated by the evidence of abnormal early enhancement with washout in the delayed phase of each imaging modality.

\section{Statistical analysis}

We statistically compared the patient profiles, tumor characteristics, and treatment procedures between the miriplatin and epirubicin groups using Fisher's exact test or unpaired $t$ test. The local control rate was calculated from the date of the TACE session to the last date on which tumor progression was documented (or the date of death of the patient). The local control rate between the two groups was compared using the Kaplan-Meier estimator with log-rank testing. Factors affecting local tumor control were first subjected to univariate analysis with the log-rank test. The parameters subjected to univariate analysis were: patient sex, age, etiology, Child-Pugh class, clinical stage, previous treatment history, tumor size, serum $\alpha$-fetoprotein level, serum des-carboxy-prothrombin level, treatment area, number of treated tumors, iodized oil dose, initial iodized oil uptake, and drug administered. Factors were considered statistically significant at $P<0.05$. Significant factors were subsequently examined by multivariate analysis using the Cox proportional hazards model.

\section{Results}

We performed targeted TACE for 56 hepatic areas of $66 \mathrm{HCC}$ nodules in 47 patients of the miriplatin group and
72 hepatic areas of 79 HCC nodules in 64 patients of the epirubicin group. The mean dose of anticancer agents used for a single TACE session was 50.5 (range, 10-100) $\mathrm{mg}$ and 17.5 (range, 5-40) mg for miriplatin and epirubicin, respectively. The patient profile, tumor characteristics, and treatment procedures in both the groups are summarized in Table 1. There were no significant differences in any of the parameters investigated between the two study groups. Although no significant difference was found, the values of the mean iodized oil dose $(P=0.060)$ and the number of treated tumors $(P=0.071)$ differed between the groups. More iodized oil was used and fewer tumors were treated in a single TACE session for the miriplatin group compared to the epirubicin group.

The median follow-up periods were 150 days (range, 25-472 days) for subjects receiving miriplatin and 340 days (range, 33-1183 days) for those receiving epirubicin. The overall recurrence rates were $39.3 \%$ (26 of 66 study lesions) and $31.6 \%$ (25 of 79 study lesions) for the miriplatin and epirubicin groups, respectively. The median periods between the TACE and local tumor progression were 122.5 days (range, 28-459 days) for 26 recurring lesions treated with miriplatin and 222 days (range, 33-808 days) for 25 recurring lesions treated with epirubicin. The local control rate was significantly higher in the epirubicin group than in the miriplatin group (log-rank test, $P<0.001$ ) (Figure 1 ).

Table I Patient profiles, tumor characteristics, and treatment procedures in the patient groups treated with miriplatin and epirubicin in the targeted chemoembolization of hepatocellular carcinoma

\begin{tabular}{|c|c|c|c|}
\hline \multirow[t]{2}{*}{ Parameters } & \multicolumn{2}{|c|}{$\begin{array}{l}\text { Chemotherapeutic } \\
\text { agent }\end{array}$} & \multirow[t]{2}{*}{$P$ value } \\
\hline & Miriplatin & Epirubicin & \\
\hline Sex (male/female) & $30 / 17$ & $43 / 21$ & 0.839 \\
\hline Mean age (years) & $70.7(35-88)$ & $70.1(48-81)$ & 0.928 \\
\hline Hepatitis (B/C/NBNC) & $7 / 30 / 10$ & $10 / 48 / 6$ & 0.233 \\
\hline Child-Pugh class (A/B) & $33 / 14$ & $46 / 18$ & 1.000 \\
\hline Clinical stage (I/II/III//VV) & $11 / 32 / 18 / 5$ & $16 / 45 / 16 / 2$ & 0.110 \\
\hline $\begin{array}{l}\text { Previous treatment } \\
\text { (primary/recurrence) }\end{array}$ & $25 / 22$ & $40 / 24$ & 0.337 \\
\hline Mean tumor size $(\mathrm{mm})$ & $17.6(6-37)$ & $17.6(7-46)$ & 0.999 \\
\hline Median AFP level ( $\mathrm{ng} / \mathrm{mL})$ & 19 (4-2098) & $12(3-6539)$ & 0.743 \\
\hline Median DCP level (mAU/mL) & $92(6-1810)$ & $93(5-4760)$ & 0.315 \\
\hline $\begin{array}{l}\text { Chemoembolized area } \\
\text { (segmental/subsegmental/distal) }\end{array}$ & $20 / 28 / 8$ & $20 / 36 / 16$ & 0.208 \\
\hline $\begin{array}{l}\text { Number of treated tumors } \\
(1 / 2 / 3)\end{array}$ & $30 / 15 / 2$ & $51 / 11 / 2$ & 0.071 \\
\hline Mean iodized oil dose $(\mathrm{mL})$ & $2.9(0.6-8.0)$ & $2.5(0.6-6.0)$ & 0.060 \\
\hline $\begin{array}{l}\text { Initial iodized oil uptake } \\
\text { (good/fair) }\end{array}$ & $58 / 8$ & $66 / 13$ & 0.488 \\
\hline
\end{tabular}

Notes: Data in brackets denote data range; no statistically significant difference was observed between the two groups for any of the parameters.

Abbreviations: AFP, $\alpha$-fetoprotein; DCP, des-carboxy-prothrombin. 
The local control rates at 6 months and 1 year were $70.7 \%$ and $44.8 \%$ for the miriplatin group and $83.4 \%$ and $69.2 \%$ for the epirubicin group, respectively. CT images of a tumor in a representative case are shown in Figure 2.

The results of the univariate analysis revealed that the Child-Pugh class $(P=0.042)$, serum $\alpha$-fetoprotein levels $(P<0.001)$, serum des-carboxy-prothrombin levels $(P=0.019)$, iodized oil dose $(P=0.005)$, initial iodized oil uptake $(P=0.036)$, and drug administered $(P<0.001)$ were the prognostic factors that significantly affected local tumor control. The results of this analysis are presented in Table 2. Further, multivariate analysis revealed that the independent factors affecting local control rate were the serum $\alpha$-fetoprotein level ( $<20 \mathrm{ng} / \mathrm{mL}$ vs $\geq 20 \mathrm{ng} / \mathrm{mL} ; P<0.001$ ), drug administered (epirubicin vs miriplatin; $P=0.002$ ), and Child-Pugh class (A vs B; $P=0.042$ ) (Table 3 ).

\section{Discussion}

Miriplatin is a novel anticancer agent that is specifically designed for the intraarterial treatment of HCC. This highly lipophilic agent is anticipated to exert antitumor effects only if it is appropriately delivered in a suspension of iodized oil and adequately accumulated in the target site over prolonged periods of time. Despite the promising results of miriplatin observed in the Phase II trials ${ }^{4,9}$ as well as the early clinical experience with this drug ${ }^{10-12}$ in TACE for treating HCC, our study demonstrated that TACE with miriplatin had significantly higher local tumor progression than that with epirubicin in patients with matched profiles, similar tumor characteristics, and treatment procedures. The local control rates of a single session of targeted lipiodol-TACE for small HCC tumors ( $<5 \mathrm{~cm}$ in diameter) were reported to be
$66.8 \%-74.4 \%$ at 1 year. $^{13,14}$ These results are comparable to the results of our study, where the local control rate of targeted TACE for the epirubicin group was $69.2 \%$. It is noteworthy that the local control rate of the miriplatin group (44.8\%) was considerably lower than that of the epirubicin group reported in our study and in previous studies.

Recently, a study from Miyayama et $\mathrm{al}^{15}$ reported that the local recurrence rate was significantly higher for miriplatin compared to epirubicin with mitomycin $\mathrm{C}$ in the lipiodolbased superselective TACE for HCC. The reported local control rates for the miriplatin group (5 months, $76.5 \%$; 10 months, $32.7 \%$ ) were in compliance with the results of our study. Since both of the studies were performed independently, the inferiority of miriplatin to epirubicin was confirmed from our study. Our study also revealed that miriplatin usage was selected as an independent factor associated with inferior local control after TACE for HCC in the multivariate Cox analysis, and the hazard ratio of using miriplatin instead of epirubicin was estimated to be $2.53(P=0.002)$. This observation has not been presented in any previously published reports.

One reason for this poor local tumor control with miriplatin could be the reduced vascular damage caused by miriplatin. Miriplatin itself causes less vascular damage than zinostatin stimalamer, ${ }^{4}$ the only oil-soluble anticancer agent approved for the intraarterial treatment of HCC in Japan. Therefore, repeated use of miriplatin for the same hepatic area is clinically feasible without inducing major vascular occlusion or arterioportal shunting. On the other hand, TACE carried out with an anthracycline anticancer agent such as epirubicin or doxorubicin has been reported to lead to a high incidence of vascular damage and occlusion of

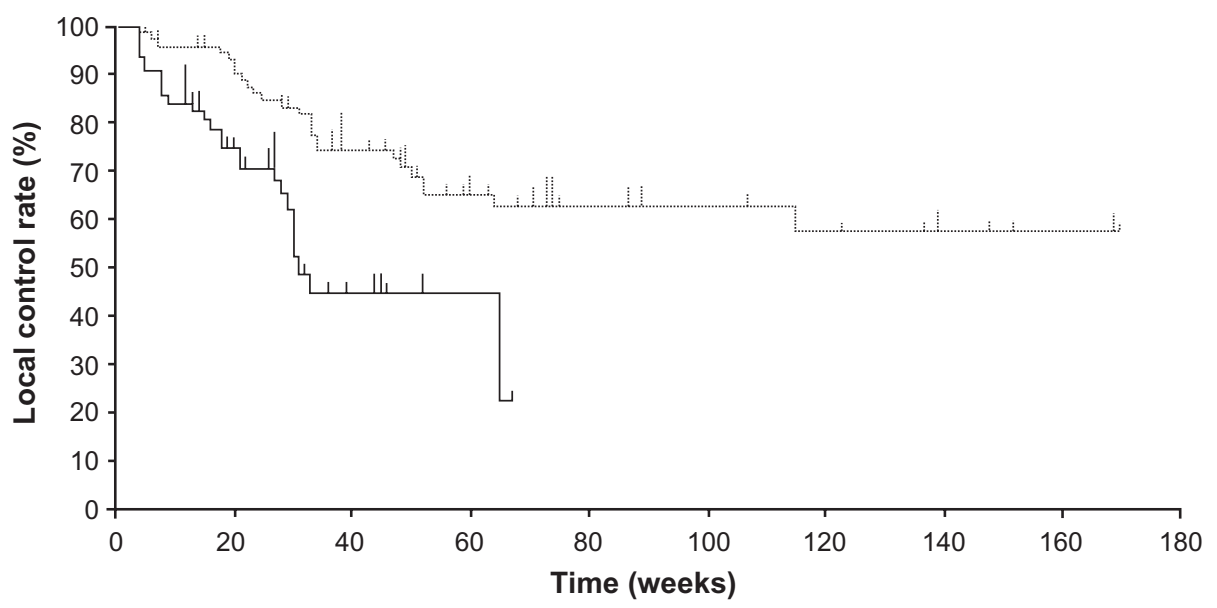

Figure I Comparison of the local control rates between the miriplatin (solid line) and epirubicin (dotted line) groups in the targeted chemoembolization of hepatocellular carcinoma.

Note: The miriplatin group showed significantly higher local tumor progression as compared to the epirubicin group $(P<0.00 \mathrm{I})$. 
A

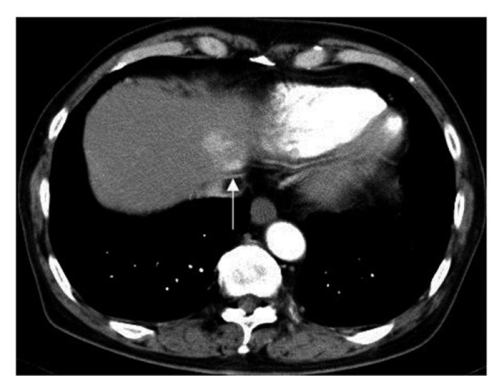

B

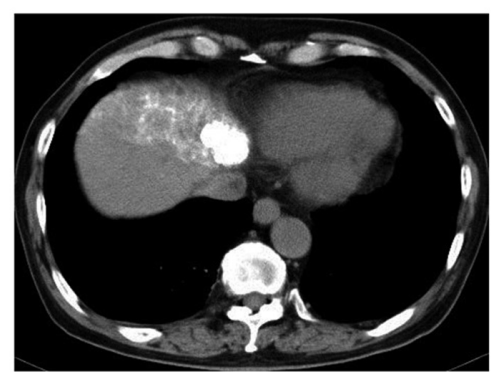

C

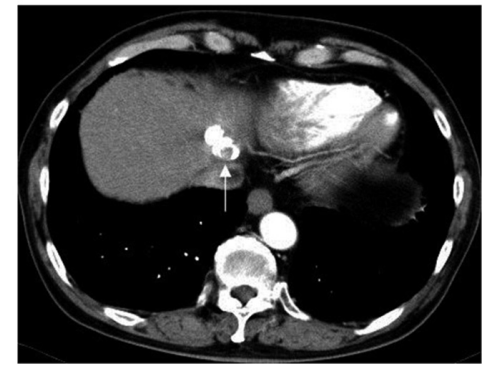

Figure 2 Hepatocellular carcinoma (diameter, $32 \mathrm{~mm}$ ) at hepatic segment II in a 78-year-old man with hepatitis $C$ who underwent chemoembolization using miriplatin. (A) Arterial phase image from dynamic contrast-enhanced computed tomography (CT) before chemoembolization delineates a well-enhanced nodular lesion located near the inferior vena cava (arrow). (B) Unenhanced CT image obtained 7 days after chemoembolization of arterial segment II + VI shows complete and dense accumulation of iodized oil within the entire lesion. (C) Arterial phase image from contrast-enhanced CT 22 weeks after chemoembolization demonstrated that the accumulated iodized oil has partially disappeared in the ventral side of the tumor where local tumor recurrence emerged (arrow).

the hepatic artery. ${ }^{16,17}$ Indeed, vascular damage is frequently observed in hepatic arteries of the treatment area after chemoembolization with epirubicin-iodized oil emulsion, hampering subsequent intra-arterial treatment by inducing extrahepatic arterial supply to the previously treated lesions. However, since some vascular damage may prevent early recanalization of tumor vessels and feeders, using miriplatin for TACE may result in earlier restoration of blood supply to the tumors and earlier washout of the iodized oil from the tumor site. To prevent early recanalization of the tumor vessels, it might be useful to add other vascular-toxic anticancer agents in the miriplatin-iodized oil suspension.

Oil suspensions have higher viscosity than water-in-oil emulsions. The viscosity of the miriplatin-iodized oil suspension may be higher than that of the epirubicin-iodized oil emulsion. In addition, we found that the oil droplets of the miriplatin-iodized oil suspension delivering to the tumor were generally much larger than those of the epirubicin-iodized oil suspension. The higher viscosity and larger chemotherapeutic droplets of the miriplatin-oil suspension may have resulted in unintentional early occlusion of narrow tumor feeders before the iodized oil could completely accumulate in the entire tumor. Although the level of iodized oil accumulation was confirmed on follow-up CT at 1 week after TACE, complete distribution of the hyperattenuated area over the treated tumor might not imply complete uptake of the iodized oil in the entire tumor. Gelatin particles used as an embolic material in this study did not obliterate tumor feeders permanently. This may be because the embolic material gradually degraded in the serum and was absorbed in the plasma over several weeks. Some contrast material injected with the gelatin particles and/or the iodized oil might have been transiently deposited in the lesions until the restoration of blood supply at the tumor site. Therefore, the therapeutic efficacy of the miriplatin-iodized oil suspension might be improved by reducing the chemotherapeutic viscosity and further fragmenting the oil droplets of the mixture, either by emulsifying the suspension with a contrast agent or by heating.

Another reason for inferior local tumor control with miriplatin may be the low serum platinum concentrations due to an excessively slow release of active platinum from the tumor site where the miriplatin-iodized oil suspension is accumulated. Miriplatin exerts an antitumor effect only when active platinum is released from the lipophilic chemical complex containing myristates as the leaving groups. In a previous study on rat hepatic tumors, only $6 \%$ of the total platinum was reported to be released into the surrounding parenchyma at 28 days after intraarterial chemoinfusion of miriplatin-iodized oil suspension in the tumors. ${ }^{6}$ Moreover, the total platinum levels in the plasma of patients receiving miriplatin at $20-120 \mathrm{mg} /$ body was approximately 300 -fold lower than those reported in previous studies of intraarterial administration of 40-100 mg cisplatin/body.,18 The maximum plasma concentration time ranged from 18-37 days for the miriplatin study, which was much longer than the 10-60 minutes observed in the cisplatin study. ${ }^{4}$ Therefore, sufficient plasma concentration of total platinum against the tumor progression is expected only when the miriplatin is retained in the target tumor for prolonged periods. The total platinum levels in the plasma and in the liver parenchyma might not have been adequate for the HCC lesions treated with miriplatin in this study. Repeated administration of miriplatin for the same lesions might increase the level of the serum platinum concentration, consequently increasing the antitumor effects of miriplatin.

In this study, one patient who received miriplatin in the TACE for HCC died of acute interstitial pneumonia 42 days after the therapy. The patient had previously been treated with 
Table 2 Univariate analysis of prognostic factors affecting local tumor progression for III patients with 145 tumors

\begin{tabular}{|c|c|c|c|c|}
\hline \multirow[t]{2}{*}{ Parameters } & \multirow[t]{2}{*}{$\begin{array}{l}\text { Number } \\
(n=145)\end{array}$} & \multicolumn{2}{|c|}{$\begin{array}{l}\text { Local control rate } \\
\text { (\%) }\end{array}$} & \multirow[t]{2}{*}{$P$ value } \\
\hline & & 6 months & I year & \\
\hline Sex & & & & 0.604 \\
\hline Male & 100 & 79.8 & 60.0 & \\
\hline Female & 45 & 72.6 & 53.8 & \\
\hline Age & & & & 0.662 \\
\hline$<70$ & 54 & 72.2 & 54.8 & \\
\hline$\geq 70$ & 91 & 79.2 & 62.2 & \\
\hline Etiology & & & & 0.602 \\
\hline $\mathrm{HBV} / \mathrm{HCV}$ & 127 & 77.8 & 58.3 & \\
\hline Others & 18 & 82.5 & 47.1 & \\
\hline Child-Pugh class & & & & $0.042 *$ \\
\hline A & 100 & 85.9 & 61.6 & \\
\hline B & 45 & 55.4 & 46.2 & \\
\hline Clinical stage & & & & 0.770 \\
\hline $\mathrm{I} / \mathrm{II}$ & 104 & 78.2 & 61.1 & \\
\hline III/IV & 41 & 75.7 & 54.8 & \\
\hline Previous treatment & & & & 0.381 \\
\hline Primary & 57 & 77.6 & 66.1 & \\
\hline Recurrence & 88 & 77.1 & 54.9 & \\
\hline Tumor size & & & & 0.088 \\
\hline$<2 \mathrm{~cm}$ & 99 & 81.4 & 63.5 & \\
\hline$\geq 2 \mathrm{~cm}$ & 46 & 69.6 & 51.0 & \\
\hline Serum AFP level & & & & $<0.00 I^{*}$ \\
\hline$<20 \mathrm{ng} / \mathrm{mL}$ & 81 & 89.1 & 72.1 & \\
\hline$\geq 20 \mathrm{ng} / \mathrm{mL}$ & 64 & 61.6 & 42.1 & \\
\hline Serum DCP level & & & & $0.019 *$ \\
\hline$<40 \mathrm{mAU} / \mathrm{mL}$ & 50 & 93.7 & 69.0 & \\
\hline$\geq 40 \mathrm{mAU} / \mathrm{mL}$ & 95 & 69.5 & 54.1 & \\
\hline Treatment area & & & & 0.371 \\
\hline Segmental & 58 & 75.0 & 52.0 & \\
\hline Subsegmental/distal & 87 & 78.9 & 63.1 & \\
\hline \multicolumn{2}{|c|}{ Number of treated tumors } & & & 0.175 \\
\hline $\mathrm{I}$ & 81 & 82.1 & 64.5 & \\
\hline $2 / 3$ & 64 & 74.3 & 55.8 & \\
\hline lodized oil dose & & & & $0.005^{*}$ \\
\hline$<3 \mathrm{~mL}$ & 79 & 88.8 & 65.0 & \\
\hline$\geq 3 \mathrm{~mL}$ & 66 & 66.0 & 53.3 & \\
\hline Initial iodized oil uptake & & & & $0.036 *$ \\
\hline Good & 121 & 80.3 & 63.0 & \\
\hline Fair & 24 & 69.6 & 37.2 & \\
\hline Drug & & & & $<0.00 I^{*}$ \\
\hline Epirubicin & 79 & 83.4 & 69.2 & \\
\hline Miriplatin & 66 & 70.7 & 44.8 & \\
\hline
\end{tabular}

Notes: *The Child-Pugh class, serum AFP level, serum DCP level, iodized oil dose, initial iodized oil uptake, and drug administered were selected as the significant prognostic factors affecting local tumor progression. Abbreviations: AFP, $\alpha$-fetoprotein; HBV, hepatitis B virus; DCP, des-carboxy-prothrombin; HCV, hepatitis $C$ virus.

epirubicin-iodized oil TACE three times for the treatment of other HCC lesions. No adverse events were documented in the previous TACE procedures with epirubicin, and the patient had no history of allergy. The patient received $58 \mathrm{mg}$ of miriplatin and $3.3 \mathrm{~mL}$ of iodized oil for two intrahepatic lesions located at hepatic segment VII and started to complain
Table 3 Multivariate analysis of prognostic factors affecting local tumor progression

\begin{tabular}{lll}
\hline Parameters & Hazard ratio & P value \\
\hline Child-Pugh class (A vs B) & $1.89(1.02-3.53)$ & $0.042^{*}$ \\
AFP $(<20 \mathrm{ng} / \mathrm{mL}$ vs $\geq 20 \mathrm{ng} / \mathrm{mL})$ & $2.96(1.66-5.26)$ & $<0.00 I^{*}$ \\
$\mathrm{DCP}(<40 \mathrm{mAU} / \mathrm{mL}$ vs $\geq 40 \mathrm{mAU} / \mathrm{mL})$ & $\mathrm{I} .6 \mathrm{I}(0.84-3.10)$ & $0.15 \mathrm{I}$ \\
lodized oil dose $(<3 \mathrm{~mL}$ vs $\geq 3 \mathrm{~mL})$ & $\mathrm{I} .57(0.83-2.97)$ & 0.162 \\
Initial iodized oil uptake (good vs fair) & $1.87(0.94-3.69)$ & 0.069 \\
Drug (epirubicin vs miriplatin) & $2.53(1.38-4.62)$ & $0.002^{*}$ \\
\hline
\end{tabular}

Notes: Data in brackets denote $95 \%$ confidence intervals; *Child-Pugh class, serum AFP level, and administered drug were the significant independent factors affecting local tumor progression.

Abbreviations: AFP, $\alpha$-fetoprotein; DCP, des-carboxy-prothrombin.

of general fatigue 3 days after the therapy. Miriplatin may have caused the acute interstitial pneumonia; however, the exact reasons remain unknown because the patient's family did not consent to an autopsy. Eosinophilia is the most frequently observed hematological toxicity of miriplatin., ${ }^{4,10,19}$ The eosinophil counts of this patient were not elevated after the therapy, but there is a possibility that an allergic reaction toward miriplatin induced acute interstitial pneumonia. No other major adverse events were observed in the present study, and the hepatic toxicities of both groups were mild and within acceptable limits.

There are several merits of using miriplatin in TACE for HCC. This third-generation platinum compound does not elicit cross-resistance to cisplatin. ${ }^{20}$ Repeated use of cisplatin often causes drug resistance and allergic reactions such as anaphylaxis. ${ }^{10,21}$ The risk of allergic reactions increases from the third session of TACE with cisplatin. ${ }^{21}$ Therefore, miriplatin can be considered as a second-line chemoembolization agent in patients who exhibit hypersensitivity or resistance to cisplatin. Cisplatin, which is a first-generation platinum compound, is associated with renal toxicity, whereas miriplatin is known to cause less renal dysfunction in comparison, ${ }^{10}$ suggesting that the latter drug is safe for use even in patients with unstable renal function without requiring excessive hydration before and after the therapy. Further, the use of miriplatin is associated with fewer adverse events as compared to cisplatin, including nausea, appetite loss, pain, anorexia, and fever. ${ }^{10}$ Combining miriplatin and other anticancer agents would potentially enhance the therapeutic efficacy of miriplatin and reduce probable adverse effects.

\section{Conclusion}

In conclusion, TACE for $\mathrm{HCC}$ using a miriplatin-iodized oil suspension resulted in inferior local tumor control compared with an epirubicin-iodized oil emulsion in patients with matched profiles, tumor characteristics, and 
treatment procedures. In the multivariate Cox proportional analysis, miriplatin usage for TACE for HCC was found to be an independent factor associated with inferior local control rate bearing a hazard ratio of 2.53 when compared to epirubicin usage. However, further investigation is required to evaluate the long-term therapeutic efficacy of miriplatin.

\section{Disclosure}

The authors report no conflicts of interest in this work.

\section{References}

1. Cammà C, Schepis F, Orlando A, et al. Transarterial chemoembolization for unresectable hepatocellular carcinoma: meta-analysis of randomized controlled trials. Radiology. 2002;224(1):47-54.

2. Llovet JM, Bruix J. Systematic review of randomized trials for unresectable hepatocellular carcinoma: chemoembolization improves survival. Hepatology. 2003;37(2):429-442.

3. Nakamura H, Hashimoto T, Oi H, Sawada S. Transcatheter oily chemoembolization of hepatocellular carcinoma. Radiology. 1989; 170(3 Pt 1):783-786.

4. Okusaka T, Okada S, Nakanishi T, Fujiyama S, Kubo Y. Phase II trial of intra-arterial chemotherapy using a novel lipophilic platinum derivative (SM-11355) in patients with hepatocellular carcinoma. Invest New Drugs. 2004;22(2):169-176.

5. Maeda M, Uchida NA, Sasaki T. Liposoluble platinum(II) complexes with antitumor activity. Jpn J Cancer Res. 1986;77(6):523-525.

6. Kishimoto S, Noguchi T, Yamaoka T, Fukushima S, Takeuchi Y. Antitumor effects of a novel lipophilic platinum complex (SM-11355) against a slowly-growing rat hepatic tumor after intra-hepatic arterial administration. Biol Pharm Bull. 2000;23(3):344-348.

7. Hanada M, Baba A, Tsutsumishita Y, et al. Intra-hepatic arterial administration with miriplatin suspended in an oily lymphographic agent inhibits the growth of tumors implanted in rat livers by inducing platinum-DNA adducts to form and massive apoptosis. Cancer Chemother Pharmacol. 2009;64(3):473-483.

8. Hanada M, Baba A, Tsutsumishita Y, Noguchi T, Yamaoka T. Intra-hepatic arterial administration with miriplatin suspended in an oily lymphographic agent inhibits the growth of human hepatoma cells orthotopically implanted in nude rats. Cancer Sci. 2009;100(1):189-194.

9. Okusaka T, Kasugai H, Ishii H. SM-11355 Japan study group. a randomized phase II trial of intra-arterial chemotherapy using a novel lipophilic platinum derivative (SM-11355) in comparison with zinostatin stimalamer in patients with hepatocellular carcinoma. J Clin Oncol. 2009;27 Suppl:abstr 4853.
10. Okabe K, Beppu T, Haraoka K, et al. Safety and short-term therapeutic effects of miriplatin-lipiodol suspension in transarterial chemoembolization (TACE) for hepatocellular carcinoma. Anticancer Res. 2011;31(9):2983-2988.

11. Imai N, Ikeda K, Seko Y, et al. Previous chemoembolization response after transcatheter arterial chemoembolization (TACE) can predict the anti-tumor effect of subsequent TACE with miriplatin in patients with recurrent hepatocellular carcinoma. Oncology. 2011;80(3-4): 188-194.

12. Imai Y, Chikayama $T$, Nakazawa $M$, et al. Usefulness of miriplatin as an anticancer agent for transcatheter arterial chemoembolization in patients with unresectable hepatocellular carcinoma. J Gastroenterol. October 6, 2011. [Epub ahead of print.]

13. Takayasu K, Muramatsu Y, Maeda T, et al. Targeted transarterial oily chemoembolization for small foci of hepatocellular carcinoma using a unified helical CT and angiography system: analysis of factors affecting local recurrence and survival rates. AJR Am J Roentgenol. 2001;176(3):681-688.

14. Miyayama S, Matsui O, Yamashiro M, et al. Ultraselective transcatheter arterial chemoembolization with a $2-\mathrm{F}$ tip microcatheter for small hepatocellular carcinomas: relationship between local tumor recurrence and visualization of the portal vein with iodized oil. JVasc Interv Radiol. 2007;18(3):365-376.

15. Miyayama S, Yamashiro M, Shibata Y, et al. Comparison of local control effects of superselective transcatheter arterial chemoembolization using epirubicin plus mitomycin $\mathrm{C}$ and miriplatin for hepatocellular carcinoma. Jpn J Radiol. January 7, 2012. [Epub ahead of print.]

16. Ono Y, Yoshimasu T, Ashikaga R, et al. Long-term results of lipiodoltranscatheter arterial embolization with cisplatin or doxorubicin for unresectable hepatocellular carcinoma. Am J Clin Oncol. 2000;23(6): 564-568.

17. Doroshow JH, Locker GY, Myers CE. Experimental animal models of adriamycincardiotoxicity. Cancer Treat Rep. 1979;63(5):855-860.

18. Shibata J, Fujiyama S, Sato T, Kishimoto S, Fukushima S, Nakano M. Hepatic arterial injection chemotherapy with cisplatin suspended in an oily lymphographic agent for hepatocellular carcinoma. Cancer. 1989;64(8):1586-1594.

19. Iwazawa J, Ohue S, Yasumasa K, Mitani T. Transarterial chemoembolization with miriplatin-lipiodol emulsion for neuroendocrine metastases of the liver. World J Radiol. 2010;2(12):468-471.

20. Hanada M, Takasu H, Kitaura M. Acquired resistance to miriplatin in rat hepatoma AH109A/MP10 is associated with increased Bcl-2 expression, leading to defects in inducing apoptosis. Oncology Rep. 2010;24(4):1011-1018.

21. Kawaoka T, Aikata H, Katamura Y, et al. Hypersensitivity reactions to transcatheter chemoembolization with cisplatin and Lipiodol suspension for unresectable hepatocellular carcinoma. J Vasc Interv Radiol. 2010 21(8):1219-1225.
Cancer Management and Research

\section{Publish your work in this journal}

Cancer Management and Research is an international, peer-reviewed open access journal focusing on cancer research and the optimal use of preventative and integrated treatment interventions to achieve improved outcomes, enhanced survival and quality of life for the cancer patient The journal welcomes original research, clinical \& epidemiological

\section{Dovepress}

studies, reviews \& evaluations, guidelines, expert opinion \& commentary, case reports \& extended reports. The manuscript management system is completely online and includes a very quick and fair peerreview system, which is all easy to use. Visit http://www.dovepress.com/ testimonials.php to read real quotes from published authors. 